\title{
Physicochemical Characterization and Solubility
}

\section{Enhancement Studies of Mebendazole Solid Dispersions in Solvent Mixtures}

\author{
Peña María Ángeles ${ }^{1}$, Escalera Begoña ${ }^{1}$, Torrado Guillermo ${ }^{1}$ and Natalini Paola ${ }^{2}$ \\ 1. Department of Biomedical Science, Facultad de Farmacia, Universidad de Alcalá, Alcalá de Henares, Madrid, Spain \\ 2. Planta Piloto de Ingeniería Química (Plapiqui-Uns-Conicet), Bahía Blanca, Argentina
}

\begin{abstract}
The objective was to obtain solid dispersion to improve the dissolution rate, solubility and oral absorption of MB (mebendazole), poor water-soluble drugs. The new formulation was characterized by DSC (differential scanning calorimetry), PXRD (powder X-ray diffraction), FT-IR (fourier transform infrared spectroscopy) and STEM (scanning transmission electron microscopy) methods. Solid dispersions of MB with polyvinylpyrrolidone K-30 (PVP K30) were prepared by solvent evaporation method. The solubility of $\mathrm{MB}$ (original powder) and that of the solid dispersions was measured at $25^{\circ} \mathrm{C}$ in ethanol-water. The aqueous solubility of MB was favoured by the presence of the polymer in solvent mixtures. Combination of solid dispersions with co-solvents increased the water solubility of MB in a larger extent that each method separately. Solubility parameter $(\delta)$ was used to relate to solubility profiles. MB and the solid dispersions show a solubility curve with a single peak at $\delta 1=30.78 \mathrm{MPa}^{1 / 2}$. Solid state characterizations indicated that the solid dispersion exist an amorphous material entrapped in polymer matrix getting highest improvement in wettability and solubility.
\end{abstract}

Key words: Mebendazole, PVP K30, solubility, solid dispersion, solubility parameter.

\section{Introduction}

One of the major problems with drugs scarcely soluble in water is its poor bioavailability after oral administration. The hidrosolubilización is the ability of a substance to be soluble in water which is very important due to the water is the main vehicle for developing pharmaceutical liquid formulations. On the other hand, the amount required of API (active pharmaceutical ingredient) with therapeutic function is frequently insoluble in water, which makes us seek alternatives to increase the solubility of the API and ensure proper dosing.

Solubilisation methods are grouped into chemical methods (forming salts, $\mathrm{pH}$ adjustment and co-solvents, as most important) and physical methods

Corresponding author: Peña María Ángeles, Ph.D. in pharmacy, professor in the Department of Biomedical Science. Email: angeles.pena@uah.es. (particle size reduction, modification of the crystalline structure, complexation, solid dispersions and micellar solubilisation). In this paper we focus on the physical method of solid dispersion for increasing the solubility. In solid dispersions, the particle size of the drugs was reduced, and the wettability and the dispersibility of the drugs were enhanced. Consequently, drug dissolution and bioavailability of hydrophobic drugs was manifestly improved. In the most recent years the solid dispersions are establish as a platform technology for a elevated number of formulation of poorly-soluble drugs such as ivacaftor, vemurafenib or itraconazole [1].

The purpose of this work mainly is to investigate the possibility of a synergic effect of co-solvents and solid dispersions for poorly water-soluble drugs like MB (mebendazole), categorized as class II compound as per the BCS (biopharmaceutics classification system), it posses very poor bioavailability, with the 
water soluble carrier PVP K30 (polyvinyl pyrrolidone K-30) to improve the aqueous solubility. Hydrophilic polymers have been widely investigated as carrier substances for solid dispersions and its high molecular size of the polymers favours the formation of solid solutions.

MB is a highly effective broad-spectrum antihelmintic indicated for the treatment of nematode infestations and the PVP K30 is a hydrophilic polymer widely employed as a carrier for preparing solid dispersions [2] due to its high aqueous solubility, excellent biocompatibility with tissues and its low toxicity [3], it was used in precise ratio (drug to carrier ratio).

The solubility studies were made in amphiprotic co-solvents such as ethanol and water, chosen to obtain binary model co-solvent systems. Models for predicting the solubility profile of the drug and the solid dispersion are also tested, Bustamante and co-workers proposed an equation to characterize curves with one maximum [4-6]:

$$
\ln \mathrm{X}_{2}=\mathrm{C}_{0}+\mathrm{C}_{1} \delta_{1}+\mathrm{C}_{2} \delta_{1}^{2}
$$

This model related the experimental solubility with the Hildebrand solubility parameter $\delta_{1}$ [7]. The properties of the drug solid phase are included as a constant into the intercept $\mathrm{C}_{0}$. The squared term $\delta_{1}{ }^{2}$ is account for self-association of the solvent through non-specific and specific interactions that decrease solubility. The linear terms are related to van der Waals $\left(\delta_{1}\right)$ solute-solvent interactions that increase solubility. The coefficients of the models are obtained from multiple linear regressions with the statistical package NCSS.

The solid dispersions were characterized by DSC (differential scanning calorimetry), PXRD (powder X-ray diffraction), FT-IR (fourier transform infrared spectroscopy) and STEM (scanning transmission electron microscopy) methods. The results suggest that the PVP K30 has a significant solubilizing effect on the drug and the drug was in the amorphous form $[8,9]$.

\section{Materials and Methods}

\subsection{Materials}

MB (methyl (5-benzoyl-1H-benzimidazol-2-yl) carbamate) (Sigma-Aldrich, Steinheim, Germany) (Fig. 1) and polyvinyl pyrrolidone K-30 (PVP K30) (1-vinyl-2-pyrrolidone polymers) (Basf, Ludwigshafen, Germany) were used as received. All these products were anhydrous, as tested with the Karl-Fisher method. Binary mixtures were prepared by volume with ethanol (UV grade, Panreac, Monplet \& Esteban, Spain) and double distilled water. All other chemicals and reagents used were of analytical grade.

\subsection{Methods}

\subsubsection{Solubility Measurements}

The solubility of MB and MB: PVP K30 solid dispersions were measured at $25^{\circ} \mathrm{C}$ in ethanol-water mixtures. $50-\mathrm{mL}$ samples containing a slight powder excess were equilibrated in a constant temperature-controlled shaking bath (Heto SH 02/100, Denmark; $\pm 0.1{ }^{\circ} \mathrm{C}$ ) at the temperature indicated. Equilibrium solubility was attained at the asymptotic region of the concentration-time profile at 48 hours. The non-dissolved drug was eliminated by filtration (Durapore membranes, $0.2 \mu \mathrm{m}$ pore size, Millipore Ibérica S.A., Spain). The drugs did not significantly adsorb onto the membranes, and the filters used were compatible with the solvents. Three samples of each solution were diluted with ethanol $96 \% \mathrm{v} / \mathrm{v}$ and assayed in a double-beam spectrophotometer (Agilent 8453, Germany) at the maximum absorption wavelength of

Mebendazole<smiles>COC(=O)Nc1nc2ccc(C(=O)c3ccccc3)cc2[nH]1</smiles>

Fig. 1 Chemical structure of mebendazole. 
the solute $(247 \mathrm{~nm})$. The concentration (molarity units) of the solutes was determined from Beer's law plots. The densities of the solutions were measured at each temperature in $10-\mathrm{mL}$ pycnometers, to convert the molar solubility into mole fraction units at each temperature.

2.2.2 Preparation of Physical Mixture by Trituration Method

MB and PVP K30 in the ratio of 1:2 were sifted through a 40 -mesh $(425 \mu \mathrm{m})$ screen, mixed together (with trituration in a pestle-mortar), and stored in a desiccated environment.

\subsubsection{Preparation of the Solid Dispersions}

The solvent method was used [10] employing acetic acid and ethanol as solvents for the drug and the carrier. The solid dispersions were prepared at a ratio of 1:2 MB-PVP K30. 0.4 grams of MB and 0.8 grams of PVP K30 were separately dissolved in $200 \mathrm{ml}$ of acetic acid and in $200 \mathrm{ml}$ of ethanol $96^{\circ}$, respectively. Both dissolutions were mixed and the organic solvent was then dried under reduced pressure using a vacuum dryer until complete evaporation at $100{ }^{\circ} \mathrm{C}$ and $45 \mathrm{rpm}$. The resultant solid dispersion was kept in a desiccator at room temperature.

Solid dispersions prepared with high proportions of PVP tend to exhibit higher drug solubility and release rate than those with high proportions of drug [2].

\subsubsection{DSC (Differential Scanning Calorimetry)} Studies

The thermograms of the original powder of the drug, the physical mixture, the polymer and the solid dispersions were obtained in equipment Mettler TA 4000, Switzerland DSC. The analysis was performed at a heating rate of $5{ }^{\circ} \mathrm{C} / \mathrm{min}$, under nitrogen flow (20 $\mathrm{mL} / \mathrm{min})$. Samples were accurately weighed $(5 \mathrm{mg})$ into aluminium sealed pans.

2.2.5 FT-IR (fourier transforms infrared spectroscopy) Studies

Fourier transform IR spectra were examined over the scanning range of 500-4000 $\mathrm{cm}^{-1}$ using a Spectrum 2000 spectrometer (Perkin Elmer, USA). The resolution was $1 \mathrm{~cm}^{-1}$. The spectra were recorded for $\mathrm{MB}$, for the crystals obtained after contact with pure solvents (re-crystallized-MB), PVP K30, the solid dispersion MB-PVP K30 (1:2) and the physical mixture MB- PVP K30 (1:2). Samples of $2 \mathrm{mg}$ were mixed with $100 \mathrm{mg}$ of potassium bromide and gently ground in a mortar. The samples were analysed from disks of about $13 \mathrm{~mm}$ diameter prepared with $\mathrm{KBr}$ and compressed in a hydrostatic press at a force of $5 \mathrm{~T}$ for 2 min.

2.2.6 PXRD (powder X-ray diffractometry) Studies

PXRD (powder X-ray diffraction) patterns were traced employing an X-ray diffractometer (Philips X Pert-MPD) for all the samples, using an Ni filter, $\mathrm{Cu}$ $\mathrm{K}(\alpha)$ radiation, a voltage of $\mathrm{kV}$, a current of $20 \mathrm{~mA}$ and a receiving slit of 0.2 inches. The samples were analyzed over a $2 \theta$ range of $5^{\circ}$ to $60^{\circ}$, with a scan step size of $0.020^{\circ}(2 \theta)$ and scan step time of 1 second.

2.2.7 STEM (scanning transmission electron microscopy) Studies

Particle morphology, size and shape were analysed by SEM. MB, PVP K30, physical mixture MB- PVP K30 (1:2) and the solid dispersion MB- PVP K30 (1:2) were placed on an aluminium sample mount, and after coating with a thin layer of gold-palladium, the samples were analysed with a zeiss electron microscopy Dsm 950. All micrographs were obtained using a magnification of $3000 \mathrm{X}$ and an accelerating voltage of $10 \mathrm{Kv}$.

\section{Results and Discussion}

Solid dispersions are not generally used in industrial products, mainly because exists the risk that during processing or storage the amorphous state may suffer crystallization [11]. That limitation includes reproducibility of physicochemical characteristics [12]. At present, different techniques are available to detect the degree of crystallinity, in this paper several of these characterisation methods have been used such as DSC (differential scanning calorimetry), PXRD (powder X-ray diffraction), FT-IR (fourier transform 
infrared spectroscopy) or STEM (scanning transmission electron microscopy).

\subsection{Solid phase Characterization}

The DSC, PXRD, STEM and FT-IR analysis confirms the formation of a solid dispersion. Fig. 2 displays the DSC profiles of MB (original powder), the polymer (PVP K30) and the solid dispersion, obtained at a heating rate of $5^{\circ} \mathrm{C} / \mathrm{min}$. The original powder shows an endothermic/exothermic transition $\left(\mathrm{T}_{\mathrm{t}}=251.6-260{ }^{\circ} \mathrm{C}, \Delta \mathrm{H}_{\mathrm{t}}=77.33 \mathrm{~kJ} / \mathrm{mol}\right)$ and a second endotherm $\left(\mathrm{T}_{\mathrm{f}}=304{ }^{\circ} \mathrm{C}, \Delta \mathrm{H}_{\mathrm{f}}=78.23 \mathrm{~kJ} / \mathrm{mol}\right)$. The endothermic peak found for PVP K30 between $\mathrm{T}_{\mathrm{g}}=150-190^{\circ} \mathrm{C}$ is due to glass transition temperature and also on the moisture content, this result was also reported by other workers [13, 14]. MB may exist under three polymorphic forms, $\mathrm{A}, \mathrm{B}$ and $\mathrm{C}$ and the thermal events found correspond to the most stable and less soluble polymorph A. The lack of a melting peak in the DSC of a solid dispersion indicates that the drug is present in an amorphous rather than a crystalline form, i.e., exist conversions of crystalline form to partial amorphous state. This is due to the amorphous form of $\mathrm{MB}$ in the solid dispersion, possibly because the carrier acts inhibiting crystallization [14].

The thermograms of MB crystallized from acetic acid and ethanol did not show new novel thermal events after equilibration with the solvents (Fig. 2), these profiles of the solid phases suggest that the solvents do not induce solid phase changes. The physical mixture, as expected, showed the thermal events from both compound, MB and PVP-K30.

The FT-IR absorption spectrum (Fig. 3) permit identify the polymorphic forms of MB because they show characteristic differences in the shape and intensity of the major absorption bands at $3370 \mathrm{~cm}^{-1}$ for the $-\mathrm{NH}$ - group and at $1730 \mathrm{~cm}^{-1}$ for the $-\mathrm{C}=\mathrm{O}$ group. In addition, a characteristic strong absorption band at 1650 $\mathrm{cm}^{-1}$ from the -CNH- group can be also used.

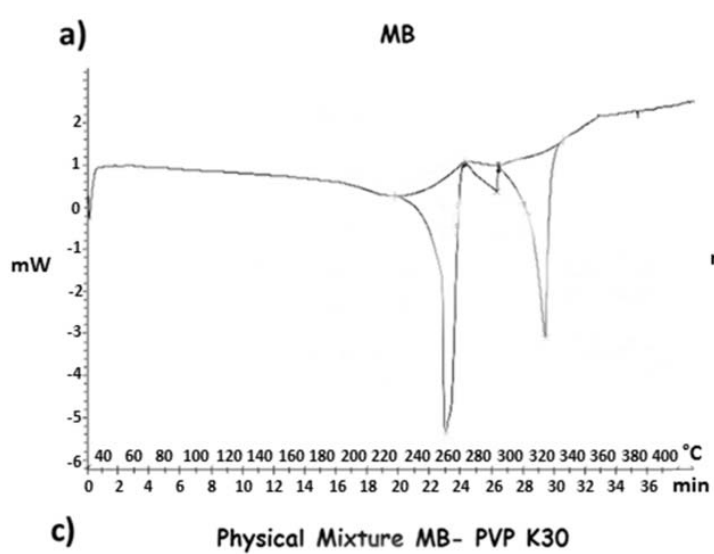

b) $\quad$ PVP K30
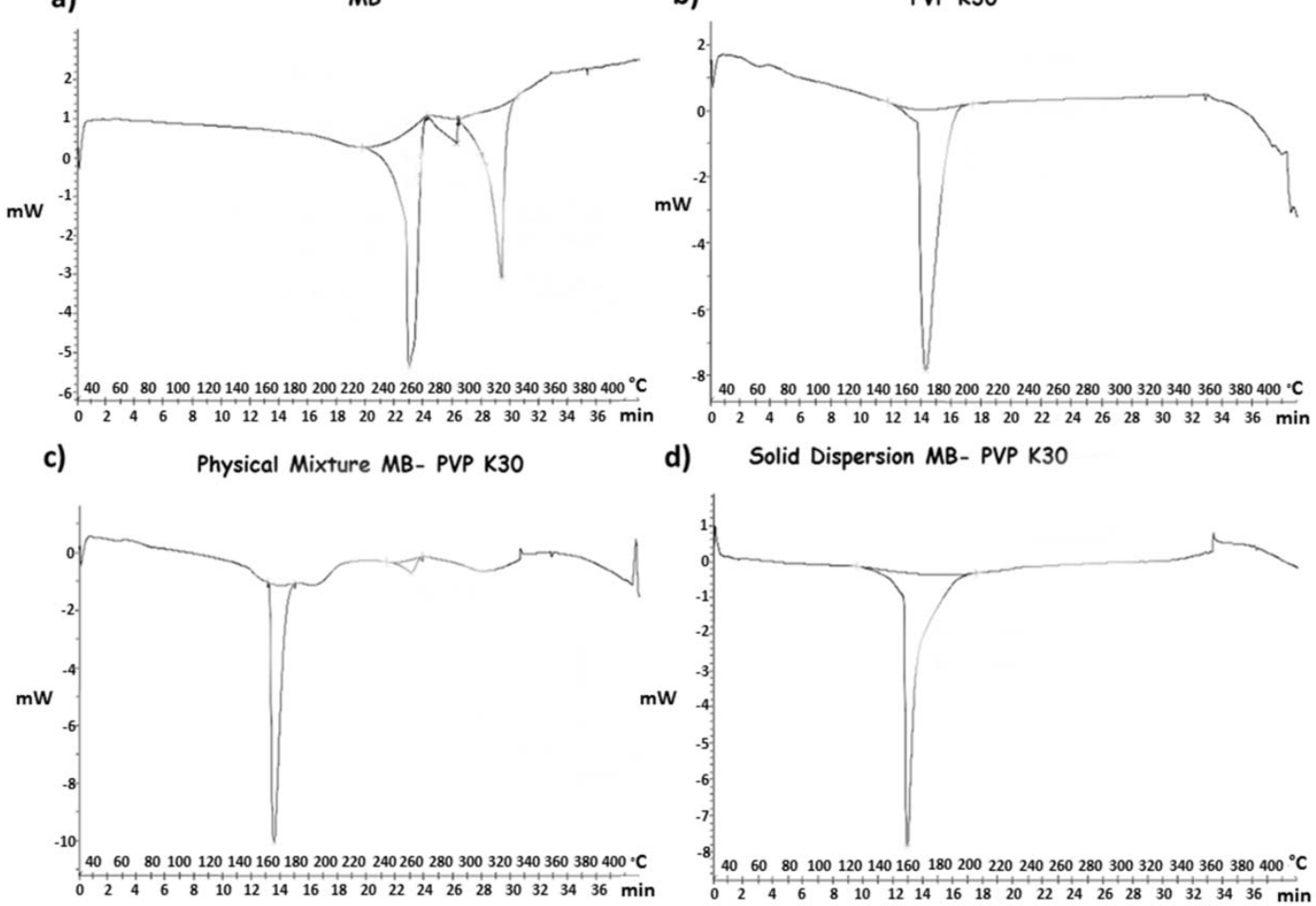

Fig. 2 DSC thermograms of MB (a), PVP K30 (b), physical mixtures MB: PVP K30 (c) and solid dispersions MB: PVP K30 (d). 
a)

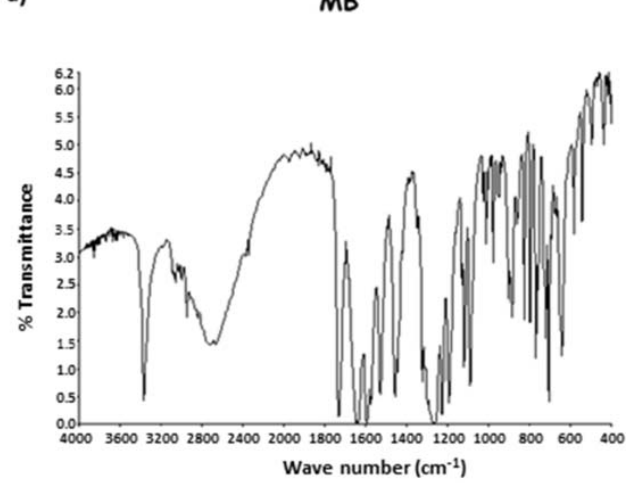

c) Physical Mixture MB- PVP K30

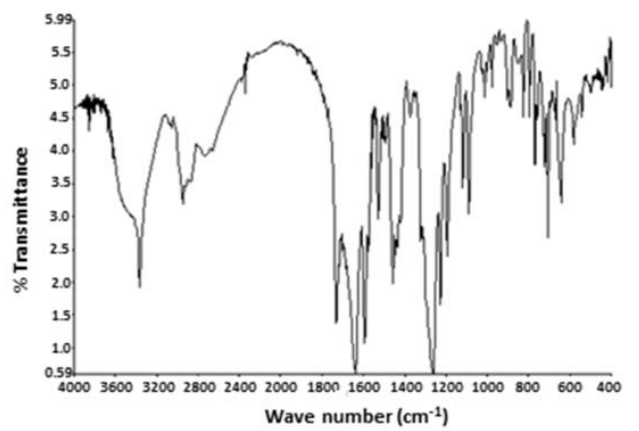

b)

PVP K30

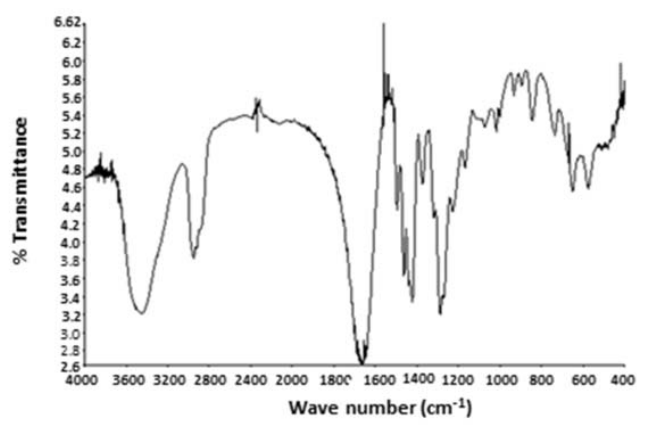

d) Solid Dispersion MB- PVP K30

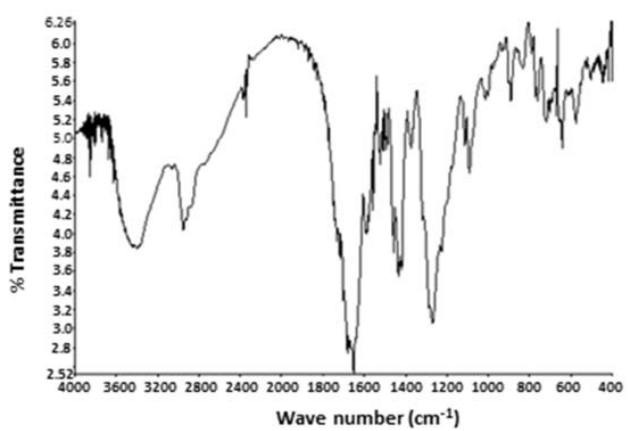

Fig. 3 FTIR curves of MB (a), PVP K30 (b), physical mixtures MB: PVP K30 (c) and solid dispersions MB: PVP K30 (d).

The most distinct peak in the IR spectrum of PVP K30 was the stretching vibration of the carbonyl group that would typically appear around 1689.53 $\mathrm{cm}^{-1}$, a broad peak at about $3000-3700 \mathrm{~cm}^{-1}$ due to $\mathrm{O}-\mathrm{H}$ stretching vibrations of absorbed water.

Through analysis by FT-IR, we have identified the polymorph $\mathrm{A}$, which characteristic absorption bands for $-\mathrm{NH} / \mathrm{C}=\mathrm{O}$ groups are $3369 / 1732 \mathrm{~cm}^{1}$. Results also shown that the graph obtained from solid dispersion presents the characteristic absorption bands from PVP $\mathrm{K}-30$, demonstrating that in the solid dispersion MB acquires amorphous characteristics.

The PXRD patterns of MB, carrier, PM and solid dispersions were given for comparative purposes and they are compared in Fig. 4. The diffraction pattern of pure drug reveals its highly crystalline nature, as indicated by the numerous distinctive peaks. The spectrum of PVP K30 was characterized by the complete absence of any diffraction peak. The lack of the numerous distinctive peaks of the drug in the PM and solid dispersions demonstrated that the drug was dissolved in solid state carrier matrix in an amorphous state. This suggests the MB present in the solid dispersion is mostly in an amorphous state with only few partially crystallized drug molecules. These assumptions were found to be in full agreement with the results presented by the DSC and FTIR studies. On the other hand, PXRD results shown that the crystal structure of pure $\mathrm{MB}$ corresponds with polymorph A $[15,16]$, data support by DSC and FTIR techniques.

STEM of MB exhibited a powder in form of needles of different sizes (Fig. 5). The physical mixture is composed of spherical PVP K30 particles surrounded by acicular MB particles. On the other hand, solid dispersions showed a clearly different appearance, being spherical particles, very similar to the PVP K30 particles. Solid state characterizations indicated that $\mathrm{MB}$ was present as an amorphous material and entrapped in polymer matrix. 
a)

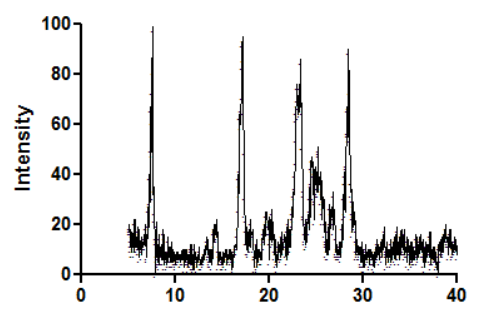

c) Physical Mixture MB- PVP K30

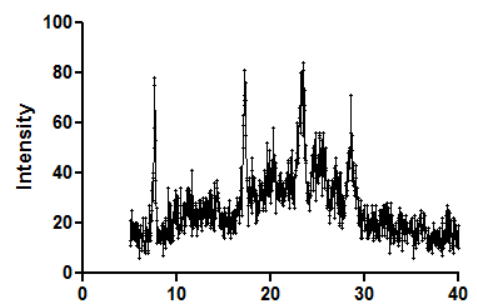

b)

PVP K30

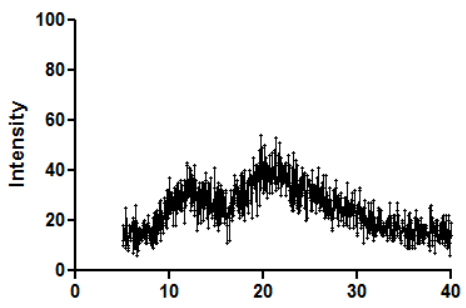

d) Solid Dispersion MB- PVP K30

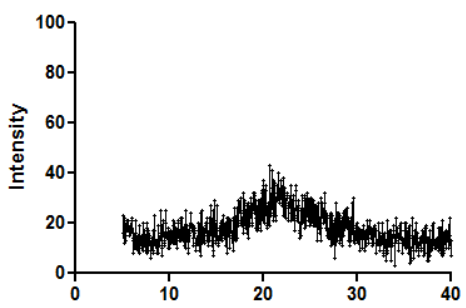

Fig. 4 PXRD patterns of MB (a), PVP K30 (b), physical mixtures MB: PVP K30 (c) and solid dispersions MB: PVP K30 (d).

a)

MB

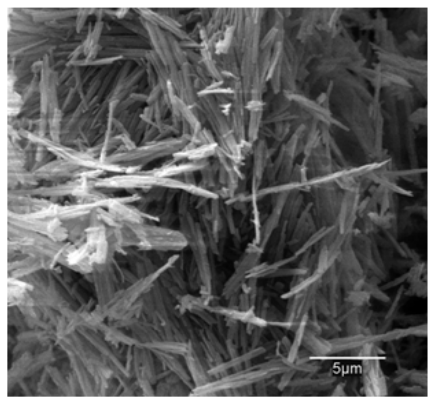

c) Physical Mixture MB- PVP K30

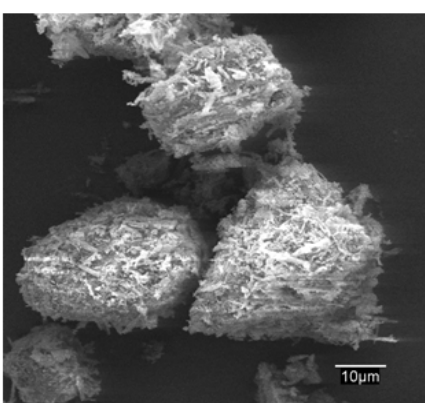

b) PVP K30

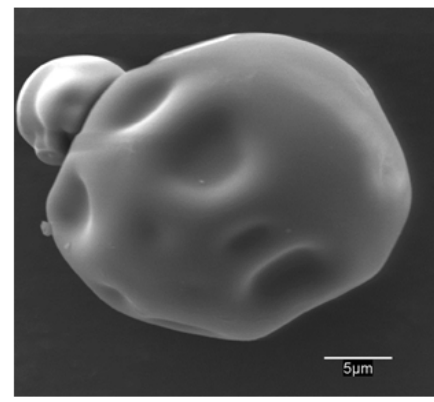

d) Solid Dispersion MBZ- PVP K30

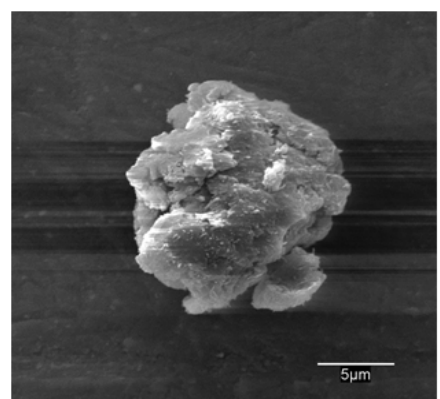

Fig. 5 STEM patterns of MB (a), PVP K30 (b), physical mixtures MB: PVP K30 (c) and solid dispersions MB: PVP K30 (d).

3.2 Solubility Profiles of the Drug and the Solid Dispersions

Table 1 lists the solubility mole fraction $\left(\mathrm{X}_{2}\right)$ of $\mathrm{MB}$ and that of the solid dispersions increases with temperature, indicating that the dissolution process is endothermic, as well the co-solvent ratio and the Hildebrand solubility parameter $\left(\delta_{1}\right)$ of the ethanol-water mixtures. The solubility of MB (Fig. 6) shows a maximum of solubility at the range included 
a)

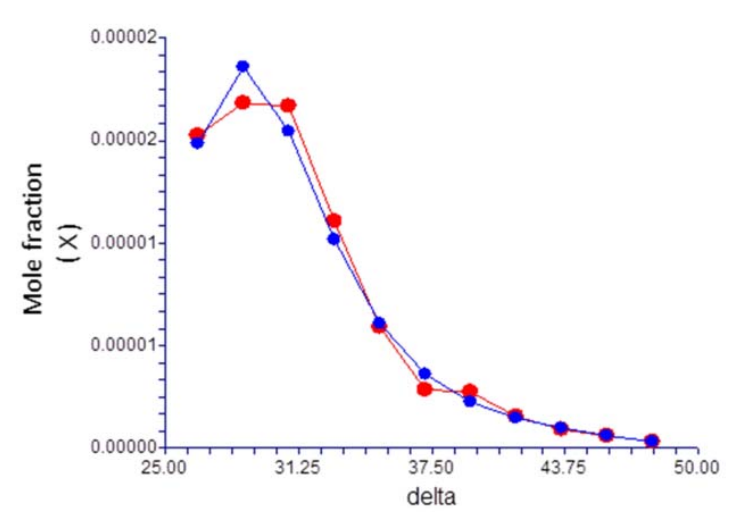

b)

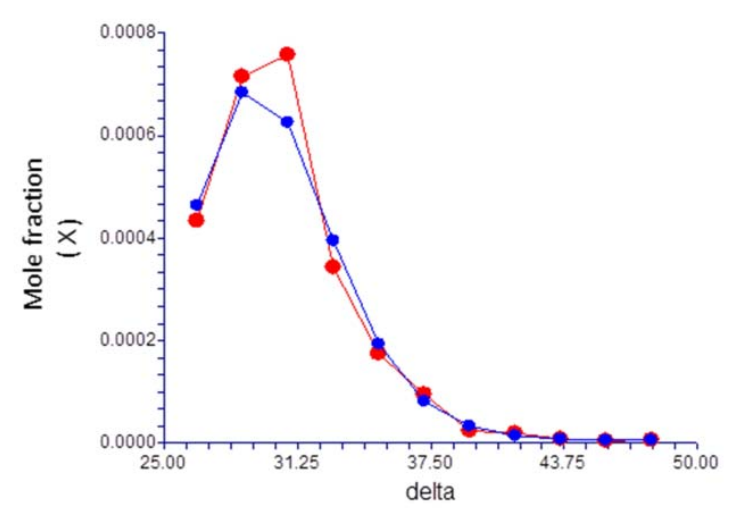

Fig. 6 Experimental $(\bullet)$ and calculated $(\bullet)$ solubility curves for the MB: PVP K30 dispersion and the MB (original powder) in ethanol-water at the $25^{\circ} \mathrm{C}$ using Equation 4 (a) and Eq. 5 (b).

between $80-95 \% \mathrm{v} / \mathrm{v}$ ethanol in water $\left(\delta_{1}=30.8-27.58\right.$ $\mathrm{MPa}^{1 / 2}$ ). In the case of the solid dispersions (Fig. 6) the solubility curve confirms a maximum $\left(\delta_{1}=30.78\right.$ $\left.\mathrm{MPa}^{1 / 2}\right)$.

The per cent solubility enhancement (\%SE) produced by the solid dispersion was calculated with Equation 2:

$$
\% \mathrm{SE}=\frac{X_{2 T}-X_{1 T}}{X_{1 T}} 100
$$

Where $X_{2 T}$ and $X_{1 T}$, are the MB: PVP K30 and MB mole experimental fractions, respectively, at a given temperature $\mathrm{T}$. The solid dispersion enhances the solubility of MB (between 87-98\%). The solubility enhancement in ethanol-water can also be fitted to a quartic-linear relationship against the solubility parameter $\left(\delta_{1}\right)$ of the ethanol-water mixture.

$$
\begin{gathered}
\% \mathrm{SE}=-941.4050+105 \delta_{1}-4.8107 \delta_{1}{ }^{2}+ \\
0.096 \delta_{1}{ }^{3}-0.0007 \delta_{1}{ }^{4} \\
\mathrm{r}^{2}=0.83 \mathrm{n}=11 \mathrm{RMSE}=2.1791
\end{gathered}
$$

Where RMSE is the root mean square error and $n$ the number of experimental cases.

\subsection{Solubility Prediction}

The experimental solubility data obtained is fitted to Bustamante model [4] using Eq. 1. An excellent correlation was obtained in both cases $\left(\mathrm{r}^{2} \geq 0.99\right)$. The predicted results obtained at $25^{\circ} \mathrm{C}$ are given in Equations 4 and 5:
MB: PVP K30 dispersions:

$$
\begin{aligned}
\ln \mathrm{X}_{2}= & -96869+7.7525 \delta_{1}-0.2159 \delta_{1}{ }^{2}+ \\
& 0.0019 \delta_{1}{ }^{3} \\
\mathrm{r}^{2}= & 0.992 \mathrm{n}=11 \mathrm{RMSE}=0.2095
\end{aligned}
$$

MB:

$$
\begin{aligned}
\ln \mathrm{X}_{2}= & -184.5656+18.929 \delta_{1}-0.7564 \delta_{1}{ }^{2}+ \\
& 0.0132 \delta_{1}{ }^{3}-0.0001 \delta_{1}{ }^{4} \\
\mathrm{r}^{2}= & 0.9936 \mathrm{n}=11 \mathrm{RMSE}=0.1466
\end{aligned}
$$

Fig. 6 illustrates the experimental and calculated solubility curves for the solid dispersion and the MB (original powder) in ethanol-water at the $25{ }^{\circ} \mathrm{C}$ (Equations 4 and 5).

\section{Conclusion}

The solid dispersion has become an established solubilisation technology for poorly water-soluble drugs. In this paper was investigated the possibility of a synergic effect of co-solvents and solid dispersions with PVP, that carrier is amongst the most frequently investigated hydrophilic polymeric carriers, to improve the aqueous solubility of MB. Solid dispersions were prepared by solvent evaporation method. The studies indicated that PVP inhibited the crystallization of drug, resulting in the amorphous state form of the drug in solid dispersion. PXRD, FT-IR, STEM and DSC results confirmed the amorphous state of drug in solid dispersion. Changes in crystallinity of the drug could explain the 
mechanisms responsible for improved dissolution.

Our results demonstrated that the use of a co-solvent as PVP in combination with the production of solid dispersions generate a synergic effect on $\mathrm{MB}$ solubility by modified the crystalline state. The increase in the solubility of $\mathrm{MB}$ becomes the formulation in a product with great potential to developing pharmaceutical liquid formulations. Also, it is expected that the new formulation obtained has an improved dissolution performance, and thus increased bioavailability. However, the dissolution studies as well as the bioavailability of this formulation should be evaluated.

\section{References}

[1] Yanbin, H., and Wei-Guo, D. 2014. "Fundamental Aspects of Solid Dispersion Technology for Poorly Soluble Drugs." Acta. Pharmaceutica Sinica B (4): $18-25$.

[2] Tantishaiyakul, V., Kaewnopparat, N., and Ingkatawornwong, S. 1996. "Properties of Solid Dispersions of Piroxicam in Polyvinylpyrrolidone K-30.” Int. J. Pharm. (143): 59-66.

[3] Caykara, T. 2004. "Solubility Parameters of Cross-linked Poly (N-vinyl-2-pyrrolidone-co-crotonic Acid) Copolymers Prepared by Gamma-Ray-Induced Polymertization Technique." J. Macromol. Sci. Appl. Chem. A. (41): 971-9.

[4] Bustamante, P., Escalera, B., Martin, A., and Selles, E. 1993. "A Modification of the Extended Hildebrand Approach to Predict the Solubility of Structurally Related Drugs in Solvent Mixtures." J. Pharm. Pharmacol. (45): 253-7.

[5] Escalera, J. B., Bustamante, P., and Martin, A. 1994. "Predicting the Solubility of Drugs in Solvent Mixtures: Multiple Solubility Maxima and the Chameleonic Effect." J. Pharm. Pharmacol. (46): 172-5.

[6] Muela, S., Escalera, B., Peña, M.A., and Bustamante, P.
2010. "Influence of Temperature on the Solubilization of Thiabendazole by Combined Action of Solid Dispersions and Co-solvents." Int. J. Pharm. (384): 93-9.

[7] Hildebrand, J. M., Prausnitz, J. M., and Scott, R. L. 1970. Regular and Related Solutions, Van Nostrand Reinhold Co. USA.

[8] Thybo, P., Kristensen, J., and Hovgaard, L. 2007. "Characterization and Physical Stability of Tolfenamic Acid-PVP K30 Solid Dispersions." Pharm. Dev. Technol. (12): 43-53.

[9] Ardianasyah, S., Nasrul, E., Rivai, H., Sahlan, E., and Zaini, E. 2015. "Physicochemical Characterization of Amorphous Solid Dispersion of Ketoprofen-Polyvinylpyrrolidone K-30." Int. J. Pharm. Pharmaceutical Sci. (2): 7-12.

[10] Habib, M. J. 2001. Pharmaceutical Solid Dispersion Technology. Technomic Publishing Go. Inc. Lancaster. Pennsylvania. USA.

[11] Chauhan, B., Shimpi, S., and Paradkar, A. 2005. "Preparation and Evaluation of Glibenclamidepolyglycolized Glycerides Solid Dispersions with Silicon Dioxide by Spray drying technique.” Eur. J. Pharm. Sci. (26): 219-30.

[12] Sameer, S., Raviraj, B., and Lalit, Y. 2011. "A Review on Solid Dispersion.” Int. J. Pharm. \& Life Sci. 2 (9): 1078-95.

[13] Leuner, C., and Dressman, J. 2000. "Improving Drug Solubility for Oral Delivery Using Solid Dispersions." Eur. J. Pharm. Biopharm. (50): 47-60.

[14] Liu, L., and Wang, X. 2007. "Improved Dissolution of Oleanolic Acid with Ternary Solid Dispersions." AAPS Pharm. Sci. Tech. (8): 1-5.

[15] Agatonovic-Kustrin, S., Glass, B. D., and Smithson, M. M. J. 2008. "Analysing the Crystal Purity of Mebendazole Raw Material and its Stability in a Suspension Formulation.” Int. J. Pharm (361): 245-50.

[16] Penna, A., Hanashiro, T., Ortega, B., Fonseca, F., Ferreira, F., and Pires, P. 2014. "Evaluation and Study of Mebendazole Polymorphs Present in Raw Materials and Tablets Available in the Brazilian Pharmaceutical Market." J. Applied Pharm. Sci. 4 (11): 1-7. 